\title{
Avaliação da lidocaína tópica como pré-medicação para a endoscopia digestiva alta em crianças
}

\author{
Evaluation of topical lidocaine spray as premedication to upper gastrointestinal endoscopy in children
}

Rodrigo Strehl Machado ${ }^{1}$, Airton Viriato ${ }^{2}$, Aurea Cristina Portorreal ${ }^{3}$

\section{RESUMO}

Objetivo: Avaliar a eficácia da lidocaína spray tópica como droga adjuvante na sedação e analgesia de crianças e adolescentes para endoscopia digestiva.

Métodos: Foram incluídos 80 pacientes ( 49 femininos e 31 masculinos, idade média $12 \pm 3$ anos), 40 no grupo placebo e 40 no grupo lidocaína. Os pacientes foram alocados aleatoriamente e um paciente de cada grupo foi excluído. Lidocaína a $10 \%$ ou placebo (ácido tânico 0,5\%) aerossol (dois jatos) foram aplicados na orofaringe antes da infusão de propofol. Os pacientes foram monitorizados durante o procedimento e após, sendo respondido questionário para avaliar odinofagia e a pré-medicação. O desfecho primário foi a dose de propofol empregada, enquanto os desfechos secundários foram incidência de complicações, tempo de sala e duração do procedimento.

Resultados: Não houve diferenças entre os grupos quanto à idade, sexo e indicação da endoscopia. A dose de propofol empregada não foi diferente nos dois grupos (grupo placebo $3,1 \pm 1,1$ e grupo lidocaína $2,9 \pm 1,3 \mathrm{mg} / \mathrm{kg} ; p=0,69$ ), mesmo quando considerada a dose bruta $(p=0,33)$. No entanto, o tempo de sala médio foi maior no grupo placebo do que no

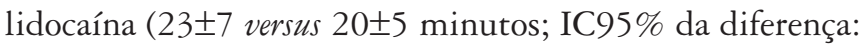
0,47-5,89 minutos, $p=0,02$ ). Não houve diferenças entre os grupos quanto à duração do procedimento, incidência de complicações e aceitação pelo paciente.

Conclusões: $\mathrm{O}$ emprego de medicação tópica anestésica em endoscopia reduz o tempo de sala sem aumentar a incidência de efeitos adversos (NCT00521703).

Palavras-chave: lidocaína; endoscopia gastrointestinal; pré-medicação.

Instituição: Hospital Infantil Cândido Fontoura (HICF), São Paulo, SP, Brasil

'Doutor em Ciências pela Universidade Federal de São Paulo (Unifesp); médico assistente do Centro de Endoscopia Digestiva e Respiratória do Hospital São Paulo (HSP-Unifesp), São Paulo, SP, Brasil

${ }^{2}$ Mestre em Promoção da Saúde pela Universidade de Franca; diretor da Divisão de Apoio Diagnóstico e Terapêutico do HICF, São Paulo, SP, Brasil ${ }^{3}$ Doutor Ciências pela Unifesp, São Paulo, SP, Brasil

\section{ABSTRACT}

Objective: This study aims to evaluate the efficacy of topical lidocaine as an adjuvant drug to sedatives in children and adolescents undergoing digestive endoscopies.

Methods: 80 patients ( 49 females and 31 males, $12 \pm 3$ years old) were randomly allocated into placebo $(n=40)$ or lidocaine group $(n=40)$. One patient was excluded from each group after allocation. Two puffs of either $10 \%$ lidocaine or placebo (tannic acid $0.5 \%$ ) were sprayed into the oropharynx before the infusion of propofol. Patients were monitored during the procedure and answered a questionnaire after the procedure to evaluate sore throat and the medicine used as pre-medication. The primary outcome measure was propofol dose. Time spent in the procedure room, in the procedure and the incidence of complications were secondary outcome measures.

Results: Demographic data (age, sex and endoscopy indication) were evenly distributed in each group. The dose of propofol was not different between patients and controls (placebo group $3.1 \pm 1.1$ and lidocaine group $2.9 \pm 1.3 \mathrm{mg} /$ $\mathrm{kg} ; p=0.69)$, even considering the total dose $(p=0.33)$. The time spent in the procedure room was longer for the placebo

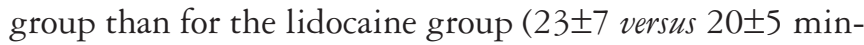
utes; 95\% CI of the difference: 0.47-5.89 minutes, $p=0.02$ ). There was no difference between groups regarding procedure duration, complications incidence and tolerability.

Conclusions: Topic anesthetic medication reduces the time spent in the procedure room without increasing the incidence of side effects (NCT00521703).

Key-words: lidocaine; endoscopy, gastrointestinal; premedication.

Endereço para correspondência:

Rodrigo Strehl Machado

Rua Pedro de Toledo 441 - Vila Clementino

CEP 04039-031 - São Paulo/SP

E-mail: rodrigo.streh|@gmail.com

Recebido em: 17/12/08

Aprovado em: 8/5/09 


\section{Introdução}

A endoscopia digestiva alta em crianças e adolescentes tem permitido uma melhor compreensão da fisiopatologia de doenças digestivas e, atualmente, é um instrumento diagnóstico e terapêutico plenamente incorporado à prática médica. Nessa faixa etária, a sedação necessária para a endoscopia digestiva é geralmente a sedação profunda, embora alguns utilizem a sedação consciente e a anestesia geral $^{(1)}$. Em crianças, o regime empregado com maior frequência é a sedação profunda ministrada por anestesista, especialmente nos grandes serviços ${ }^{(2)}$. O propofol é amplamente empregado nesse contexto, sendo um agente anestésico e sedativo de curta duração, que permite rápida recuperação após o exame e possui como principais efeitos adversos dor no local da infusão, apnéia transitória e hipotensão ${ }^{(2)}$. Estudo comparativo recente demonstrou que o propofol melhora a qualidade do exame endoscópico, permitindo melhor visualização dos marcos anatômicos e, possivelmente, reduzindo a necessidade de repetição do exame ${ }^{(3)}$. A droga é pelo menos tão segura quanto a combinação de meperidina e midazolam na sedação para endoscopia digestiva alta em crianças, com a vantagem de requerer menos frequentemente a contenção do paciente, ter rápido início de ação e pronta recuperação ${ }^{(4)}$. Além disto, a droga possui ação sinérgica com o midazolam, requerendo menores doses quando usado em associação ${ }^{(5)}$.

A eficácia e a segurança do propofol lançam dúvida sobre a necessidade do uso de anestesia tópica com lidocaína como medicação pré-anestésica em crianças. A lidocaína é um anestésico local que interage com um receptor situado no canal de sódio da fibra nervosa, tornando lenta a despolarização da membrana. É o anestésico tópico faríngeo mais empregado em nosso meio para a realização de exame endoscópico. $\mathrm{O}$ emprego de anestésico tópico faríngeo melhora a tolerância do procedimento e facilita a sua realização em pacientes não sedados, quando comparado ao uso de placebo ${ }^{(6)}$. Estudo recente mostrou que a lidocaína tópica, comparada ao placebo e à não-medicação, reduziu o aumento da frequência cardíaca e da pressão arterial sistólica ocorrido no início do exame $e^{(7)}$. Apesar de os pacientes avaliados serem adultos submetidos à sedação consciente com midazolam ou placebo, tal efeito poderia levar à diminuição da dose necessária de medicação sedativa, com consequente redução dos custos e complicações a ela relacionados. Além disso, a anestesia tópica apresenta algumas desvantagens, pois, em pacientes sedados, há dúvidas quanto à sua tolerabilidade, sendo relatado que alguns a consideram moderadamente desconfortável, pois é irritativa e deixa um gosto amargo na boca ${ }^{(8)}$. Em crianças, esse efeito poderia potencialmente aumentar a dose necessária de medicação sedativa. Ademais, o seu uso pode elevar o custo do exame se a ele não corresponder a uma redução do tempo gasto em cada procedimento, além de diminuição do tempo de ocupação da sala de procedimento e da dose de medicação sedativa. Por fim, efeitos adversos podem ocorrer após a anestesia tópica da orofaringe ${ }^{(9,10)}$. Além disso, a anestesia tópica da faringe interfere com a deglutição e o fechamento da glote, constituindo fator de risco para aspiração e consequente pneumonia ${ }^{(10)}$. Ainda não está claro se isso poderia levar a uma maior incidência de complicações respiratórias (hipoxemia, hipoventilação) durante o procedimento em pacientes com sedação profunda.

Não existem estudos para avaliar a utilidade da prémedicação com lidocaína em pacientes pediátricos recebendo sedação profunda com propofol para a realização de endoscopia digestiva. Assim, este estudo tem como objetivo avaliar a eficácia da anestesia faríngea tópica com lidocaína em reduzir a dose necessária de propofol para sedação suficiente de crianças e adolescentes submetidos à endoscopia digestiva alta.

\section{Métodos}

O estudo foi delineado como um ensaio clínico aleatorizado, duplo-cego, controlado por placebo. Foram incluídos 80 pacientes encaminhados ao serviço de endoscopia do Hospital Infantil Cândido Fontoura para realizar endoscopia digestiva alta em regime ambulatorial, sendo 49 do sexo feminino (61\%), com idade variando entre 8 e 19,9 anos (média 12,5 anos, mediana 12 anos, desvio padrão 2,7). Os pacientes foram incluídos prospectiva e consecutivamente, sendo considerados critérios de inclusão a idade entre $8 \mathrm{e}$ 18 anos e o peso superior a 30kg. Foram excluídos aqueles com comorbidades e doenças neuropsiquiátricas que necessitavam de tratamento contínuo, as crianças e adolescentes com sondas nasogátricas, com alergia conhecida à medicação (lidocaína) ou cujo pai ou responsável não tenha consentido em participar do estudo.

Após convite para participação voluntária no estudo e assinatura de Termo de Consentimento Livre e Esclarecido pelo responsável, os pacientes foram distribuídos aleatoriamente em dois grupos, sendo 40 pacientes para o grupo A e 40 pacientes para o grupo B. Dois pacientes foram excluídos após a distribuição aleatória, sendo um do grupo A ( 9 anos, feminina) por agitação psicomotora que impediu o prosseguimento do procedimento, e outro do grupo B (11 anos, 
masculino) por retirada do consentimento. O estudo foi aprovado pelo Comitê de Ética em Pesquisa da Universidade Federal de São Paulo, após anuência da instituição em que o estudo foi realizado, e foi condicionado à inexistência de custos para a instituição.

Os pacientes alocados para o grupo A receberam quatro jatos de spray de placebo (ácido tânico $0,5 \%$ ) em orofaringe, enquanto os pacientes do grupo B receberam quatro jatos de spray da medicação (lidocaína 10\%). Os grupos foram definidos por sorteio e os códigos de randomização foram guardados por uma terceira parte, sendo que o médico endoscopista, o médico anestesista e o paciente (ou responsável) não estavam cientes da droga empregada em cada caso individual até concluída a análise estatística dos dados. Os códigos seriam quebrados em caso de efeito adverso significativo que necessitasse intervenção, fato não ocorrido. O protocolo foi registrado no sítio Clinical Trials.gov (http://www.clinicaltrials.gov) sob a identificação NCT00521703.

A endoscopia digestiva alta foi realizada conforme a rotina do serviço. Todos os pacientes foram sedados com propofol (dose a critério do anestesista), sendo usada outra droga a critério do anestesista (midazolam e/ou fentanil) e monitorizados com cardioscópio e oximetria de pulso durante todo o procedimento. Todos os pacientes receberam oxigênio (2L/ $\mathrm{min}$ ) por cateter nasal durante o procedimento. $\mathrm{O}$ aparelho de endoscopia empregado foi o gastrofibroscópio Fujinon FG 100-PE. A ocorrência de taquicardia (FC>120bpm), bradicardia ( $\mathrm{FC}<50 \mathrm{bpm}$ ) e hipoxemia (Sp O2 <94\%) foram anotadas, assim como sua duração. $\mathrm{O}$ tempo de sala foi definido como o tempo decorrido entre a entrada do paciente na sala e a sua saída. O tempo de procedimento foi definido como a duração da endoscopia. Após o procedimento, os pacientes foram inquiridos sobre a ansiedade antes do procedimento, sua avaliação do spray empregado e dor de garganta após o procedimento. Essas perguntas foram feitas no momento da alta e, para cada uma delas, foi empregada uma escala visual de 10 pontos $^{(11)}$.

A randomização foi feita através de tabela gerada por computador, sendo usado sítio da internet (http://www. randomization.com). As tabelas de randomização foram geradas a cada 20 pacientes incluídos, em blocos de quatro. O desfecho primário foi a dose de propofol empregada, enquanto os desfechos secundários foram a incidência de complicações, tempo de sala e duração do procedimento. Considerando que uma diferença clinicamente significativa entre os dois grupos seria igual ou superior a 0,5 desvios padrão (DP), com nível de significância de 0,05 e poder de $85 \%$, a amostra foi definida em 80 pacientes em cada grupo (total de 160 pacientes). Durante o estudo, os dados foram analisados a cada 20 pacientes incluídos para verificar diferença significativa entre os grupos quanto a variáveis de segurança (tempo de sala, tempo de procedimento, presença de bradicardia, taquicardia e hipoxemia), situação em que o estudo seria interrompido. Entretanto, o estudo foi interrompido porque houve diferença significativa entre os dois grupos quanto ao tempo de sala.

As variáveis quantitativas contínuas foram descritas por suas médias e DP, ou por sua mediana e distância interquartil (DIQ), conforme o caso. As variáveis qualitativas foram comparadas de acordo com o grupo de alocação por meio do qui-quadrado de Pearson ou teste exato de Fisher, quando necessário. As variáveis quantitativas foram avaliadas quanto à normalidade por análise do histograma. As variáveis de distribuição normal foram comparadas pelo teste $t$ de Student e, para as variáveis com distribuição diferente da normal, foi empregado o teste de Mann-Whitney ${ }^{(12)}$. Em todos os testes foi considerado significativo um valor de $p<0,05$. A análise estatística foi feita por meio do programa $\mathrm{R}$ ( $\mathrm{R}$ Development Core Team, disponível em http://cran.r-project.org) em ambiente Linux (versão 2.6, distribuição Ubuntu 7.10).

\section{Resultados}

A indicação mais comum de endoscopia foi a dor epigástrica, seguida de dor abdominal. Os dados demográficos de ambos os grupos estão dispostos na Tabela 1. Não houve diferenças entre os dois grupos quanto a sexo, idade, história de endoscopia prévia e indicação do procedimento.

A dose média de propofol empregada não foi diferente nos dois grupos $(3,1 \pm 1,1 \mathrm{mg} / \mathrm{kg}$ no grupo A e $2,9 \pm 1,3 \mathrm{mg} / \mathrm{kg}$ no grupo $\mathrm{B} ; p=0,697)$, mesmo quando considerada a dose bruta (136,9 $\pm 46,0 \mathrm{mg}$ no grupo A e 126,4 $\pm 49,1 \mathrm{mg}$ no grupo B; diferença $=10,5 \mathrm{mg}$; IC95\%: $-10,9$ a 32; $p=0,33)$. Em apenas $12(15 \%)$ pacientes o propofol foi usado como droga única; em 23 (29\%), o midazolam e o fentanil foram associados; em 2 (3\%), o fentanil foi aplicado; e em 41 ( $52 \%$ ), o midazolam. O midazolam foi usado em 64 (82\%) pacientes: $33 / 39(55 \%)$ do grupo A e 31/39 (79\%) do grupo B ( $p=0,55)$. O fentanil foi empregado em 25 (32\%), dos quais $12 / 39$ (31\%) eram do grupo A e 13/39 (33\%) do grupo B ( $p=0,81)$. O tempo de sala médio foi de $21 \pm 6$ minutos, sendo significativamente maior no grupo A (23 \pm 7 minutos) do que no grupo B (20 \pm 5 minutos; IC95\% da diferença $=0,5-5,9$; teste $t, p=0,02$ ) (Figura 1). O tempo de procedimento mediano foi 5 minutos 
Tabela 1 - Dados demográficos

\begin{tabular}{lccc}
\hline & Grupo A (n=39) & Grupo B (n=39) & Valor de $\boldsymbol{p}$ \\
\hline Sexo feminino & $25(64 \%)$ & $23(59 \%)$ & 0,64 \\
Idade & $12,5 \pm 2,7$ & $12,6 \pm 2,8$ & 0,93 \\
Endoscopia prévia & $9(23 \%)$ & $23(29 \%)$ & 0,21 \\
Indicação da endoscopia & & & 0,90 \\
Epigastralgia & $24(61 \%)$ & $22(56 \%)$ & \\
Dor abdominal & $8(20 \%)$ & $8(20 \%)$ & \\
Controle de tratamento & $4(10 \%)$ & $4(10 \%)$ & \\
Vômitos & 0 & $2(5 \%)$ & \\
Dor retroesternal & 0 & $2(5 \%)$ & \\
Hipertensão portal & $1(3 \%)$ & $1(3 \%)$ & \\
Outras & $2(5 \%)$ & 0 & \\
\hline
\end{tabular}

(DIQ 4-5) no grupo A e 4 minutos (DIQ 4-5) no grupo B (teste de Mann-Whitney, $p=0,27$ ).

A maioria dos pacientes avaliou o spray de forma positiva (54/78 - 70\%), 11/78 (14\%) avaliaram-no de forma neutra e 12/78 (16\%) de forma negativa, sendo a avaliação negativa igualmente frequente nos dois grupos. Odinofagia foi relatada por 22 pacientes (29\%), sendo 12 (31\%) do grupo A e 10 (26\%) do grupo B $(p=0,60)$.

Não houve efeitos colaterais diretamente associados à medicação, assim como não ocorreu bradicardia. Houve 27 (35\%) episódios de hipoxemia (12/39 no grupo A e 15/39 no grupo $\mathrm{B} ; p=0,47)$ e 6 episódios ( $8 \%$ ) de taquicardia (4/39 no grupo A, 2/39 grupo $\mathrm{B} ; p=0,67)$. Os episódios de hipoxemia

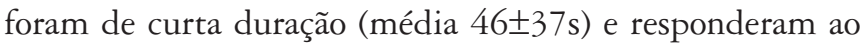
aumento do fluxo de oxigênio e estímulo do paciente, não sendo necessária a ventilação em nenhum caso. A ocorrência de hipoxemia não foi associada à dose de propofol, midazolam ou fentanil empregada.

\section{Discussão}

O presente estudo é o primeiro ensaio clínico aleatorizado, duplo-cego e placebo controlado sobre a eficácia da anestesia tópica faríngea em crianças e demonstrou que seu emprego é bem tolerado, não está associado à maior incidência de efeitos colaterais e levou à redução do tempo de sala do procedimento. No entanto, o emprego da lidocaína não se associou com menor dose de medicação sedativa ou diminuição do tempo do procedimento. O tempo de sala neste estudo se refere basicamente à soma entre o preparo do paciente (instalação da monitorização, aplicação do spray), o procedimento e o tempo de recuperação (até recuperar a consciência $)^{(13,14)}$. Sabendo-se que a duração do procedimento foi homogênea, assim como o preparo, possivelmente o menor

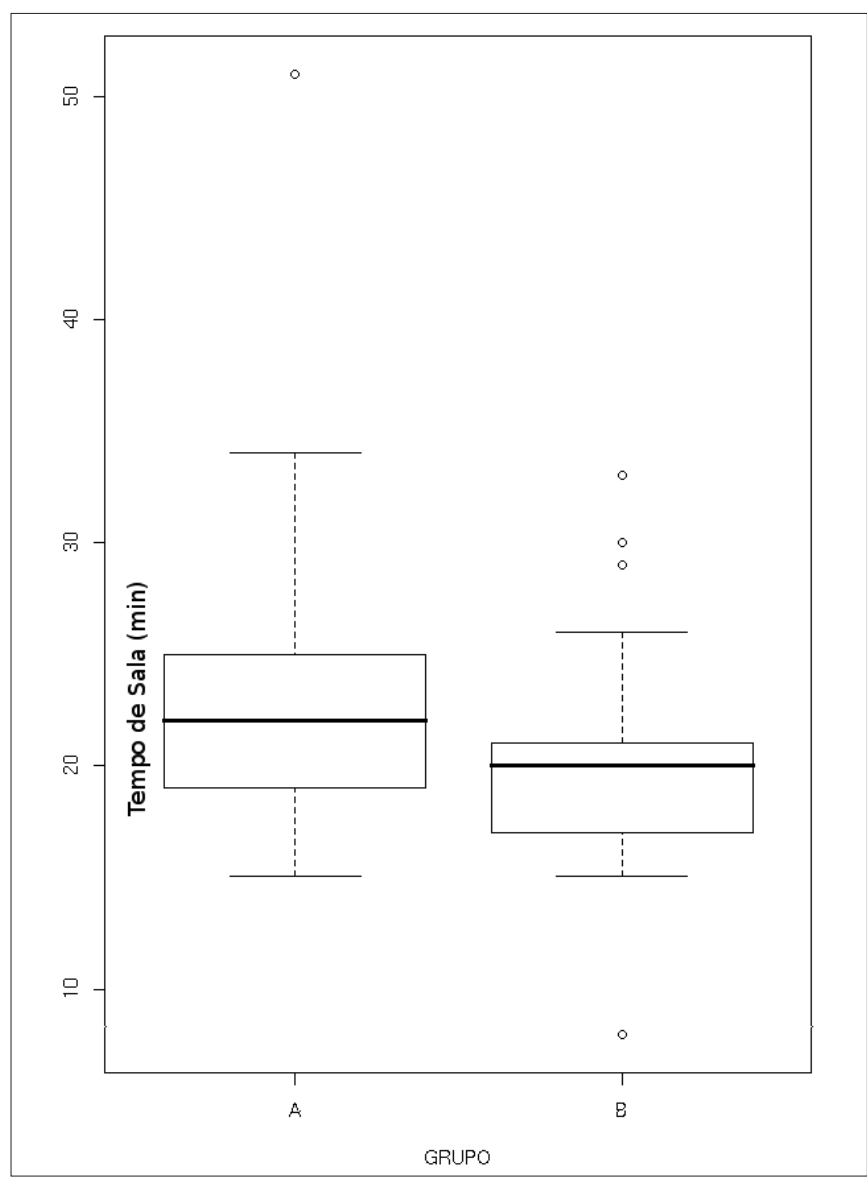

Figura 1 - Duração da permanência dos pacientes na sala de procedimento

tempo de sala tenha sido consequência de menor tempo para recuperação da consciência. Embora a diferença tenha sido pequena entre os dois grupos, ela foi relevante e representaria, ao fim de um turno de trabalho, um paciente examinado a mais. Esse achado levou à interrupção do estudo porque a sua continuidade implicaria custos para a instituição em que o estudo foi realizado. 
Não foram observados efeitos adversos associados à lidocaína spray. No entanto, os pacientes foram observados por tempo reduzido. Se, de um lado, isto levou a uma taxa mínima de perdas, de outro não foi possível avaliar efeitos colaterais tardios da medicação. Estudo realizado no Reino Unido identificou risco significativo de pneumonia aspirativa em pacientes que haviam utilizado anestésico tópico para realizar a endoscopia digestiva ${ }^{(10)}$. Embora o anestésico tópico possa interferir com mecanismos neuromusculares fisiológicos de proteção da via aérea, facilitando a aspiração e a instalação da pneumonia, os achados daquele estudo não foram reproduzidos por outros grupos e os anestésicos tópicos continuam a ser recomendados ${ }^{(11)}$.

A incidência de hipoxemia no estudo (35\%) pode ser considerada alta, sendo possíveis diversas explicações. $\mathrm{O}$ ponto de corte para definir hipoxemia foi elevado (94\%) e muitos dos pacientes não apresentaram saturação inferior a 90\%. Recentemente, estudo sobre a segurança do propofol, quando usado em endoscopia com pacientes adultos, relatou 2,3\% de hipoxemia, definida como saturação inferior a 90\%, taxa similar à relatada $(1,5 \%)$ por grupo multicêntrico, que avaliou prospectivamente a segurança da endoscopia digestiva em crianças $^{(15,16)}$. Por outro lado, pesquisa recente que analisou o uso do propofol associado à ketamina ou fentanil para endoscopia digestiva descreveu taxa de hipoxemia (definida como saturação de oxigênio inferior a 90\%) similar à descrita no presente trabalho (33\%), sendo mais alta no grupo que usou fentanil e propofol $(50 \%)^{(17)}$. No presente estudo não foi verificada relação entre o uso de midazolam e/ou fentanil e hipoxemia, mas apenas em 12 pacientes o propofol foi empregado como droga única, pois o estudo não foi desenhado para avaliar essa associação. De modo geral, a incidência de hipoxemia em crianças submetidas à endoscopia digestiva nas quais foi empregado propofol é maior do que em pacientes adultos, possivelmente devido à necessidade de sedação mais profunda ${ }^{(18)}$.

Há dúvidas quanto à vantagem do uso de anestesia tópica faríngea em endoscopia digestiva. $\mathrm{Na}$ presente pesquisa apenas $12(16 \%)$ pacientes relataram que o emprego do spray antes da endoscopia foi desconfortável, seis pacientes de cada grupo. Estudo similar realizado com adultos verificou que a tolerância à endoscopia digestiva sob sedação consciente foi similar entre aqueles tratados ou não com anestésico tópico, mas a facilidade para realizar o exame, conforme avaliação do médico examinador, foi menor nos pacientes tratados ${ }^{(8)}$. Recentemente, uma meta-análise revisou cinco ensaios clínicos controlados para determinar se a anestesia tópica faríngea melhora a tolerância do paciente à endoscopia digestiva alta e verificou haver associação entre boa tolerância à endoscopia e o emprego de anestesia tópica, tanto pela avaliação do paciente quanto do médico ${ }^{(19)}$. Não há estudos similares em crianças, mas observamos boa tolerabilidade da prémedicação tópica entre os pacientes, não havendo diferenças entre medicação e placebo.

A diferença de dose empregada de propofol entre os dois grupos não foi significativa. O uso do propofol é geralmente associado ao de outras drogas sedativas, pois seu emprego isolado pode necessitar de maior dosagem e, consequentemente, maior tempo de recuperação em adultos e $\operatorname{crianças~}^{(18,20)}$. Em crianças, por exemplo, a associação com midazolam ou fentanil leva à menor necessidade de doses suplementares da medicação e reduz a incidência de efeitos adversos ${ }^{(21)}$. Devido a isso, foi facultado ao médico anestesista o emprego de outras drogas e seu impacto na pesquisa foi avaliado estatisticamente, não sendo significativo. Não há estudos verificando o impacto do uso de anestesia tópica faríngea sobre a dose de propofol empregada na sedação e este estudo sugere que tal efeito, se existe, é pequeno. No entanto, pode ter sido clinicamente significativo, pois o menor tempo de sala observado no grupo tratado é consequência do menor tempo para a recuperação de consciência.

Em conclusão, o emprego de lidocaína tópica como prémedicação em endoscopia digestiva na infância não permite a redução da dose necessária de propofol para a adequada sedação do paciente. No entanto, seu uso foi associado a uma significativa e relevante redução do tempo de sala do procedimento, sem aumentar a incidência de efeitos adversos e complicações.

\section{Agradecimentos}

Agradecemos aos anestesistas Luís Faria e Lair Valio pela colaboração na realização deste estudo. 


\section{Referências bibliográficas}

1. Michaud L. Sedation for diagnostic upper gastrointestinal endoscopy: a survey of the Francophone Pediatric Hepatology, Gastroenterology, and Nutrition Group. Endoscopy 2005;37:167-70.

2. Barbi E, Petaros P, Badina L, Pahor T, Giuseppin I, Biasotto E et al. Deep sedation with propofol for upper gastrointestinal endoscopy in children, administered by specially trained pediatricians: a prospective case series with emphasis on side effects. Endoscopy 2006;38:368-75.

3. Meining A, Semmler V, Kassem AM, Sander R, Frankenberger U, Burzin M et al. The effect of sedation on the quality of upper gastrointestinal endoscopy: an investigator-blinded, randomized study comparing propofol with midazolam. Endoscopy 2007;39:345-9.

4. Khoshoo V, Thoppil D, Landry L, Brown S, Ross G. Propofol versus midazolam plus meperidine for sedation during ambulatory esophagogastroduodenoscopy. J Pediatr Gastroenterol Nutr 2003;37:146-9.

5. Sinfontes de Karam L, Díaz A, Trejo C, Palacios J, Toro M. Sedación profunda con propofol vs. propofol-midazolam para estudios endoscópicos en la edad pediátrica. GEN 2004;58:27-32.

6. Campo R, Brullet E, Montserrat A, Calvet X, Rivero E, Brotons C. Topical pharyngeal anesthesia improves tolerance of upper gastrointestinal endoscopy: a randomized double-blind study. Endoscopy 1995;27: 659-64.

7. Ristikankare M, Julkunen R, Heikkinen M, Mattila M, Laitinen T, Wang SX et al. Sedation, topical pharyngeal anesthesia and cardiorespiratory safety during gastroscopy. J Clin Gastroenterol 2006;40:899-905.

8. Davis DE, Jones MP, Kubik CM. Topical pharyngeal anesthesia does not improve upper gastrointestinal endoscopy in conscious sedated patients. Am J Gastroenterol 1999;94:1853-6.

9. Dahshan A, Donovan GK. Severe methemoglobinemia complicating topical benzocaine use during endoscopy in a toddler: a case report and review of the literature. Pediatrics 2006;117:e806-9.

10. Quine MA, Bell GD, McCloy RF, Charlton JE, Devlin HB, Hopkins A. Prospective audit of upper gastrointestinal endoscopy in two regions of England: safety, staffing, and sedation methods. Gut 1995;36:462-7.
11. Shaoul R, Higaze H, Lavy A. Evaluation of topical pharyngeal anaesthesia by benzocaine lozenge for upper endoscopy. Aliment Pharmacol Ther 2006;24:687-94.

12. Vittinghoff E, Glidden DV, Shiboski SC, McCulloch CE. Regression methods in biostatistics: linear, logistic, survival, and repeated measures models. New York: Springer; 2005.

13. Abraham NS, Fallone CA, Mayrand S, Huang J, Wieczorek P, Barkun AN. Sedation versus no sedation in the performance of diagnostic upper gastrointestinal endoscopy: a Canadian randomized controlled cost-outcome study. Am J Gastroenterol 2004;99:1692-9.

14. Montes RG, Bohn RA. Deep sedation with inhaled sevoflurane for pediatric outpatient gastrointestinal endoscopy. J Pediatr Gastroenterol Nutr 2000;31:41-6.

15. Külling D, Orlandi M, Inauen W. Propofol sedation during endoscopic procedures: how much staff and monitoring are necessary? Gastrointest Endosc 2007;66:443-9.

16. Thakkar K, El-Serag HB, Mattek N, Gilger MA. Complications of pediatric EGD: a 4-year experience in PEDS-CORI. Gastrointest Endosc 2007;65:213-21.

17. Tosun Z, Aksu R, Guler G, Aliye E, Aynur A, Duran A et al. Propofol-ketamine vs propofol-fentanyl for sedation during pediatric upper gastrointestinal endoscopy. Paediatr Anaesth 2007;17:983-8.

18. Paspatis GA, Charoniti I, Manolaraki M, Vardas E, Papanikolaou N, Anastasiafou A et al. Synergistic sedation with oral midazolam as a premedication and intravenous propofol versus intravenous propofol alone in upper gastrointestinal endoscopies in children: a prospective, randomized study. J Pediatr Gastroenterol Nutr 2006;43:195-9.

19. Evans LT, Saberi S, Kim HM, Elta GH, Schoenfeld P. Pharyngeal anesthesia during sedated EGDs: is "the spray" beneficial? A meta-analysis and systematic review. Gastrointest Endosc 2006;63:761-6.

20. VanNatta ME, Rex DK. Propofol alone titrated to deep sedation versus propofo in combination with opioids and/or benzodiazepines and titrated to moderate sedation for colonoscopy. Am J Gastroenterol 2006;101:2209-17.

21. Disma N, Astuto M, Rizzo G, Rosano G, Naso P, Aprile G et al. Propofol sedation with fentanyl or midazolam during oesophagogastroduodenoscopy in children. Eur J Anaesthesiol 2005;22:848-52. 